\title{
Erratum to: Physical exercise programs in CKD: lights, shades and perspectives
}

Filippo Aucella ${ }^{1}$ - Yuri Battaglia ${ }^{2} \cdot$ Vincenzo Bellizzi $^{3}$. Davide Bolignano $^{4} \cdot$ Alessandro Capitanini $^{5}$.

Adamasco Cupisti $^{6}$

Published online: 27 May 2015

(C) Italian Society of Nephrology 2015

Erratum to: J Nephrol (2015) 28:143-150

DOI 10.1007/s40620-014-0169-6

The title is incorrect.

The correct title is "Physical exercise programs in CKD: lights, shades and perspectives".

The online version of the original article can be found under doi:10.1007/s40620-014-0169-6.

Filippo Aucella

f.aucella@operapadrepio.it

1 Department of Nephrology and Dialysis, Scientific Institut for Research and Health Care "Casa Sollievo della Sofferenza" IRCCS, Viale Cappuccini n. 1, 71013 San Giovanni Rotondo, FG, Italy

2 Department of Nephrology, University-Hospital St. Anna, Ferrara, Italy

3 Division of Nephrology, Dialysis and Renal Transplantation, University Hospital "San Giovanni di Dio e Ruggi d'Aragona", Salerno, Italy

4 CNR-Institute of Clinical Physiology, Reggio Calabria, Italy

5 Nephrology Unit, Pescia-Pistoia Hospital, Pescia, Italy

6 Department of Clinical and Experimental Medicine, University of Pisa, Pisa, Italy 